\title{
Perceptual transparency in neon color spreading displays
}

\author{
VEBJØRN EKROLL and FRANZ FAUL \\ Christian-Albrechts-Universität, Kiel, Germany
}

\begin{abstract}
In neon color spreading displays, both a color illusion and perceptual transparency can be seen. In this study, we investigated the color conditions for the perception of transparency in such displays. It was found that the data are very well accounted for by a generalization of Metelli's (1970) episcotister model of balanced perceptual transparency to tristimulus values. This additive model correctly predicted which combinations of colors would lead to optimal impressions of transparency. Color combinations deviating slightly from the additive model also looked transparent, but less convincingly so.
\end{abstract}

The phenomenon of neon color spreading was first noticed by Varin (1971) and van Tuijl (1975). This illusion owes its name to the apparent diffusion of color, which can be seen in Figure 1. This particular configuration consists of a black and blue grid on a physically white background. Despite this fact, the background close to the blue parts of the grid looks bluish. Other interesting phenomenal facts pertaining to this illusion (see Figure 1) are that the embedded part seems to be desaturated, as compared with the same part viewed in isolation, and that one has the (sometimes rather vague) impression of a transparent layer (Bressan, Mingolla, Spillmann, \& Watanabe, 1997; Varin, 1971) covering the region of the subjective color spread.

Different explanations have been proposed for the subjective color spreading. Bressan (1993a), for instance, proposed that color spreading is due to ordinary assimilation of the von Bezold type and that "Bezold-type assimilation, when taking place within a surface that is further seen as transparent, turns into the neon spreading effect" (p. 360). In accordance with this, she suggested that neon color spreading and Bezold-type assimilation "should be parsimoniously interpreted as the same basic phenomenon" (p. 361).

Anderson (1997) proposed a different approach, which "asserts that the geometric and luminance relationships of contour junctions induce illusory transparency and lightness percepts by causing a phenomenal scission of a homogeneous luminance into multiple con-

This work was made possible by Grant DFG FA 425/1-1 from the Deutsche Forschungsgemeinschaft to F.F. We thank Bart Anderson, Jürgen Golz, Reinhard Niederée, Eike Richter, Charles M. M. de Weert, Daniel Wollschläger, and several anonymous reviewers for valuable comments on previous drafts of this manuscript. We are also indebted to Rainer Mausfeld for his continuous support and advice and to Adrienne Huggard and Margaret Ribeiro-Nelson for helping us out with the English. Correspondence concerning this article should be addressed to V. Ekroll, Institut für Psychologie der Christian-Albrechts-Universität zu Kiel, Olshausenstr. 40, 24118 Kiel, Germany (e-mail: vekroll@psychologie.uni-kiel.de). tributions" (p. 419). The scission "is assumed to cause changes in perceived lightness and/or surface opacity." By the term scission, he refers both to a perceptual decomposition of local luminance into a transparent layer and an underlying surface and to a perceptual decomposition into the reflectance of a surface and the prevailing illumination. This approach may also be considered theoretically parsimonious, since it links the brightness illusion observable in neon color spreading displays to mechanisms of transparency perception. A further advantage of this account is that it seems to be applicable to other well-known brightness and color illusions, such as the Munker-White illusion and Benary's ${ }^{1}$ illusion (Anderson, 1997).

We have been pursuing an approach that bears some resemblance to that of Anderson (1997) and is, in part, inspired by it. In particular, we consider the idea of connecting the color and/or brightness illusions in neon color spreading to the perceptual scission of local proximal color information into a transparent layer component and a background component to be a promising one. A difference between our approach and that of Anderson is that he made the working assumption "that the achromatic contrast ... is the primary determinant of scission," whereas we have been adopting the working hypothesis that chromatic properties also play an important role. This seemed natural to us, given the fact that this has already been found to be true by several authors (Chen \& D'Zmura, 1998; Da Pos, 1989; D’Zmura, Colantoni, Knoblauch, \& Laget, 1997; Faul, 1996, 1997) for four-region transparency displays, originally studied by Metelli (1970). Related to this is a further difference between our approaches. Anderson's model does not rest on Metelli's episcotister metaphor but makes similar predictions for the special cases of isochromatic and achromatic stimuli. In contrast, we have chosen to think in terms of the episcotister model. An important reason for this is that it can easily be generalized and applied to three-dimensional color codes (Chen \& D'Zmura, 1998; Da Pos, 1989; D'Zmura et al., 1997; Faul, 1997). 

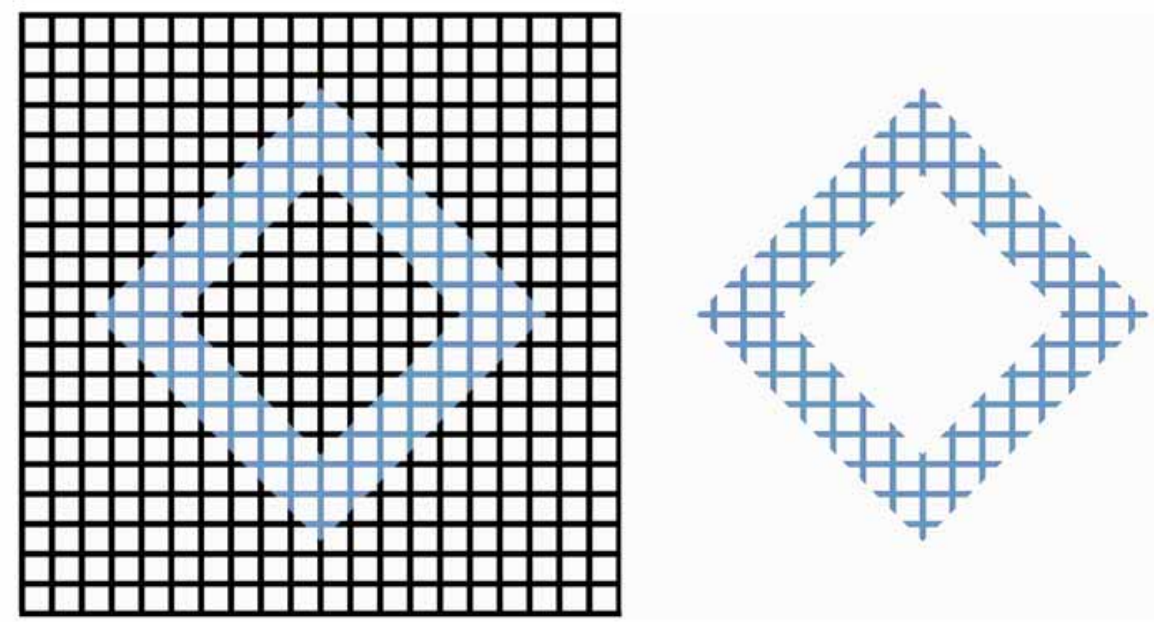

Figure 1. Neon color spreading as it was presented by van Tuijl (1975). Note that the blue color on the left and on the right is physically the same.

An episcotister is simply a disk with an open sector. Since the very beginning of experimental psychology, this apparatus has been a preferred device for modifying the effects of light reaching the eye from a given object in lawful ways. Let $\alpha$ be the size of the open sector relative to the entire disk - that is, $0<\alpha<1$. If we have an episcotister $E$ rotating quickly in front of a bipartite field consisting of regions $A$ and $B$, then perceptually, four re- gions, $A, B, P$, and $Q$, are given (see Figure 2). It can be shown with reference to Talbot's (1934) law of color fusion that, for this situation, the equations

$$
f(P)=\alpha f(A)+(1-\alpha) f(E)
$$

and

$$
f(Q)=\alpha f(B)+(1-\alpha) f(E)
$$
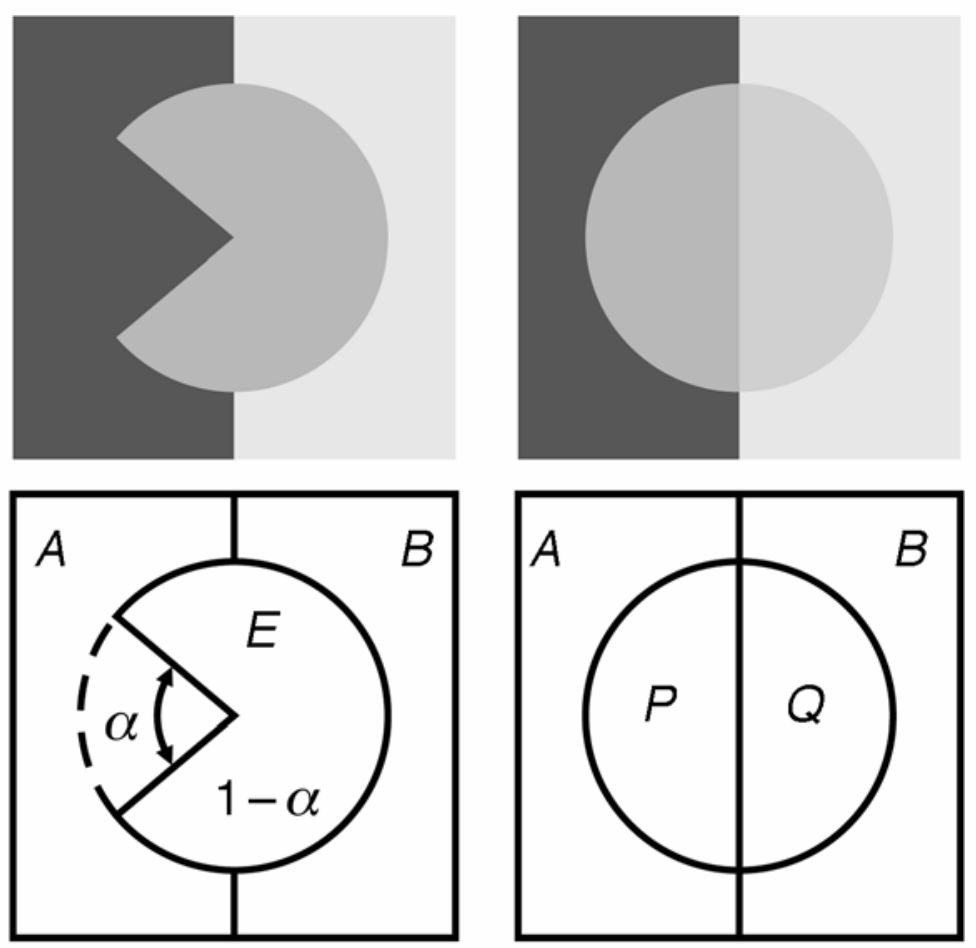

Figure 2. The episcotister model (Metelli, 1970). On the left side, the episcotister is depicted; on the right side is the perceptual impression resulting from the rotation of the episcotister. 
hold, where $f(X)$ may be taken to be the light reflected from region $X$ or any linear function of this light, such as, for instance, luminance, $\ell$. For the sake of correctness, it should be noted that the expressions on the left side of the equations are only effective lights (or, for $f:=\ell$, effective luminances) - that is, the temporally alternating lights, reflected from these regions, have the same effect on the photoreceptors as a steady light of the intensity and spectral composition given by $f(P)$ or $f(Q)$, respectively.

Solving for $\alpha$ and choosing $f:=\ell$ yields

$$
\alpha=\frac{\ell(P)-\ell(Q)}{\ell(A)-\ell(B)} .
$$

Since the transmittance $\alpha$ of the episcotister is subject to the natural restrictions $0<\alpha$ and $\alpha<1$, it follows that

$$
\ell(P)<\ell(Q) \Leftrightarrow \ell(A)<\ell(B)
$$

and

$$
|\ell(P)-\ell(Q)|<|\ell(A)-\ell(B)|,
$$

respectively. In summary, if a mosaic of colors, as displayed in Figure 2, is due to the presence of an episcotister, then Equation 4, stating that the episcotister cannot reverse the direction of contrast, must hold, as well as Equation 5, stating that the effect of an episcotister can only be to reduce (or, in the limiting case of $\alpha=1$, preserve) the amount of contrast.

According to the episcotister model, perceptual transparency should be observed whenever these two conditions of preservation of contrast direction (Equation 4) and reduction of contrast (Equation 5) are met. The conditions for perceptual scission described by Anderson (1997) are consistent with, and in many respects equivalent to, these two conditions.

It has been shown, however, that the perception of transparency does not depend only on the luminance relations of the stimuli, but also on chromatic relations (Chen \& D'Zmura, 1998; Da Pos, 1989; D'Zmura et al., 1997; Faul, 1997). These relations may be modeled with the episcotister model, since it can easily be extended to tristimulus values. The cone excitation $\phi_{L}$ of the longwavelength-sensitive L-cones is a linear function of light and may, thus, be substituted for $f$ in Equations 1 and 2. The same holds for the cone excitations $\phi_{M}$ and $\phi_{S}$ of the M-cones and the S-cones. This yields

$$
\vec{\phi}(P)=\alpha \vec{\phi}(A)+(1-\alpha) \vec{\phi}(E)
$$

and

$$
\vec{\phi}(Q)=\alpha \vec{\phi}(B)+(1-\alpha) \vec{\phi}(E),
$$

with $\vec{\phi}(X):=\left[\phi_{L}(X), \phi_{M}(X), \phi_{S}(X)\right]^{t}$. Although the models of Chen and D'Zmura (1998), Da Pos (1989), D'Zmura et al. (1997), and Faul (1997) differ in some respects, this natural extension of the episcotister model is a core element in all of them. This model, which we will refer to as the strict additive model, leads to more restrictive con- ditions for the perception of transparency than do Equations 4 and 5. For instance, if one solves Equations 6 and 7 for $\alpha$ componentwise, one gets

$$
\alpha_{i}=\frac{\phi_{i}(P)-\phi_{i}(Q)}{\phi_{i}(A)-\phi_{i}(B)}
$$

for $i=L, M, S$. Equations 6 and 7 imply that $\alpha_{L}=\alpha_{M}=\alpha_{S}$. Since the episcotister transmittance $\alpha_{i}$ may be said to reflect the degree of contrast reduction for the cone type $i$, this means that the degree of contrast reduction, computed separately for each class of cones, must be equal for all three cone classes. The strict additive model describes a very simple geometrical structure in three-dimensional color space. The color shifts caused by the episcotisterthat is, the difference vectors $\vec{\phi}(P)-\vec{\phi}(A)$ and $\vec{\phi}(Q)-$ $\vec{\phi}(B)$ - must converge to a common point, $\vec{\phi}(E)$, if the region $P \cup Q$ is to be seen as a homogeneous transparent layer. ${ }^{2}$ Consequently, the model has been called a convergence model by D'Zmura et al. (1997).

The strict additive model describes available data for the perception of transparency in four-region stimuli rather well (Chen \& D'Zmura, 1998; Faul, 1997). Thus, it seems reasonable to take this model as a point of departure for studying the perception of transparency in neon color spreading displays.

A difference between the four-region stimuli originally studied by Metelli (1970) and neon color spreading displays is that the latter, in purely proximal terms, have only three differently colored regions. ${ }^{3}$ This may be appreciated in Figures 3 and 4, which depict a well-known neon brightness/color spreading display called the modified Ehrenstein figure (Redies \& Spillmann, 1981; Redies, Spillmann, \& Kunz, 1984). In proximal terms, the color of region $Q$ is equal to the color of region $B$. In perceptual terms, however, the color of region $Q$ is different from the color of region $B$. This illusory color of region $Q$ is the color spreading effect. Besides color information, figural cues also seem to play a critical role. In neon color displays, they give rise to a virtual contour that separates regions $Q$ and $B$ (de Weert \& van Kruysbergen, 1987; Kanizsa, 1980; Schumann, 1900). An as-

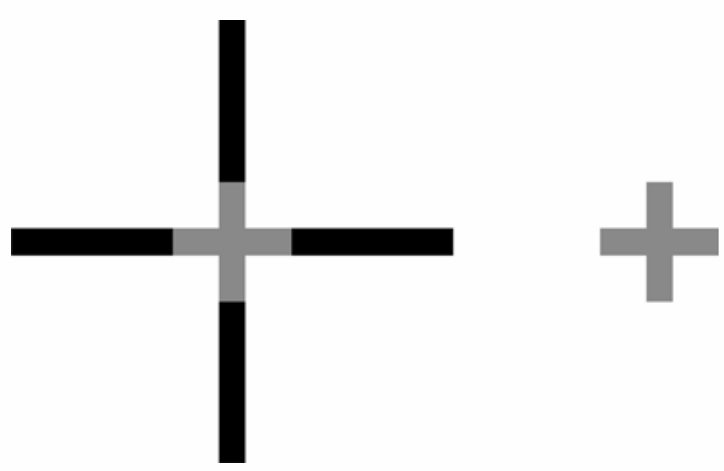

Figure 3. An achromatic version of the modified Ehrenstein figure. 

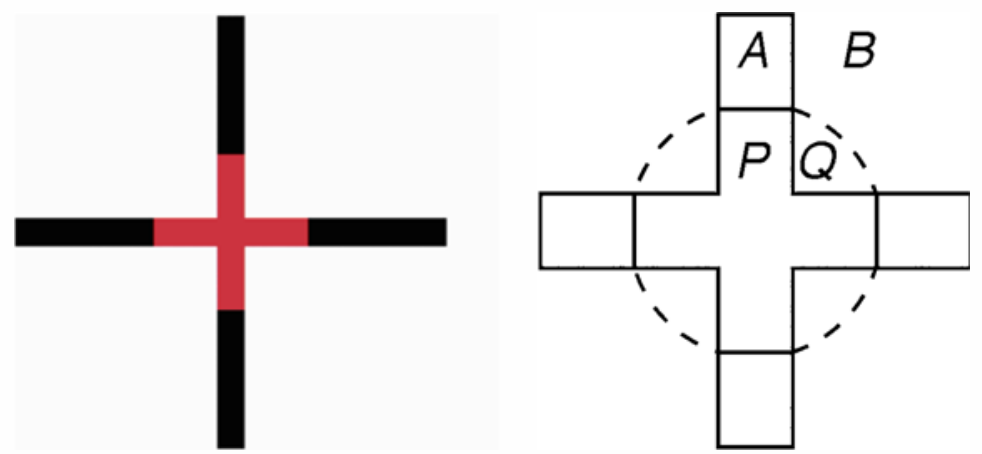

Figure 4. Left: a chromatic version of the modified Ehrenstein figure. Right: schematic depiction of the four perceptually defined regions.
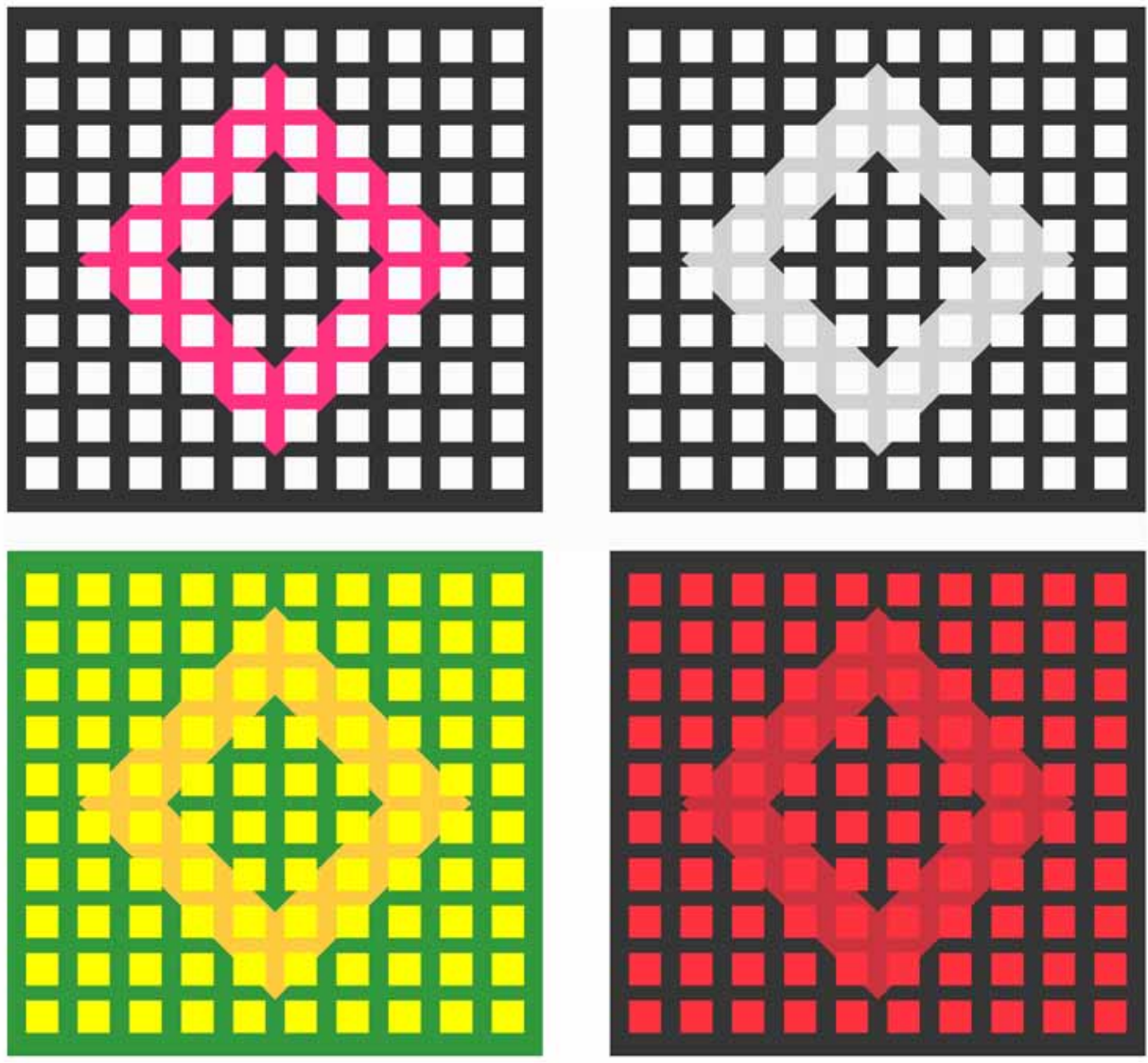

Figure 5. Four instances of neon color spreading stimuli (van Tuijl, 1975). The left configurations have a combination of colors that is often used for demonstrations of the neon color spreading effect. These color combinations do not fit the strict additive model. Still, one has the impression of transparency. However, the impression is rather vague, and it is not balanced-that is, one does not see the grid in its original color through a homogeneous transparent layer. The other two color combinations fit the strict additive model as far as reproduction allows it. In these configurations, the impression of transparency is more convincing and balanced.

sumption implicit to our approach is that the separation of regions $Q$ and $B$ is due to figural cues.

The model of Anderson (1997) is equally applicable to this configuration and to four-surface stimuli, because of its focus on contours instead of regions. However, it is also possible to apply the strict additive model (Equations 6 and 7) to this configuration, a possibility that was clearly spelled out and investigated by Watanabe and Ca- 
vanagh (1993) and discussed by Grossberg (1987, p. 132). Since in such configurations, $\vec{\phi}(Q)=\vec{\phi}(B)$ always holds, the strict additive model may be rewritten as

$$
\vec{\phi}(P)=\alpha \vec{\phi}(A)+(1-\alpha) \vec{\phi}(B) .
$$

This is a model of balanced transparency and predicts that region $P \cup Q$ should look transparent whenever $0<\alpha<$ 1 . The geometrical interpretation of this condition is that the tristimulus vector $\vec{\phi}(P)$ lies on the line segment connecting the tristimulus vectors $\vec{\phi}(A)$ and $\vec{\phi}(B)$ in tristimulus color space. In the achromatic case, this means that $\ell(A)<\ell(P)<\ell(B)$ or $\ell(B)<\ell(P)<\ell(A)$. Since these relations are invariant with respect to monotonic transformations of $\ell$, the same predictions follow if one assumes that perceived lightness, as opposed to luminance, is the relevant variable, which has been proposed by Beck and co-workers (Beck \& Ivry, 1988; Beck, Prazdny, \& Ivry, 1984). It is also apparent that this model makes exactly the same predictions as Anderson's (1997) model for achromatic (and isochromatic) stimuli-namely, that the region $P \cup Q$ should look transparent whenever the luminance of $P$ is between the luminances of $A$ and $B$. In general, however, the present model is clearly more restrictive.

Before we describe the experiments performed to test the present model, some clarifying comments may be required. If one regards the modified Ehrenstein figure depicted on the left side of Figure 4, it becomes clear that this stimulus fails to satisfy Equation 9. If one assigns the tristimulus values $\vec{\phi}(A):=(0,0,0)^{t}$ to the outer cross $A$, which is black, and the tristimulus values $\vec{\phi}(B):=(1,1,1)^{t}$ to the background $B$, which is white, the model predicts that the tristimulus values of the inner cross $P$ must be $\vec{\phi}(B):=(x, x, x)^{t}$ with $0<x<1$ (which has to be some shade of gray) if region $P \cup Q$ is to be perceived as a homogeneous transparent layer. Since $P$ is not gray, the stimulus does not fit the strict additive model. It looks transparent, however. One may be tempted to conclude from this that the proposed model is wrong as it stands and that it is a waste of time performing experiments to test it.

However, this conclusion is warranted only if the strict additive model is taken to describe all stimuli that look transparent. In the present approach, we hypothesize that the strict additive model describes only stimuli that lead to an impression of balanced transparency that is optimally convincing. All impressions of transparency are not equally compelling. This point may be appreciated in Figure 5. In all four configurations, one is prone to say that one has an impression of transparency. However, most observers would probably agree that the impressions of balanced transparency in the left configurations are less evident than in the right configurations. This subtle but, in our opinion, important distinction may get lost in investigations in which subjects are asked to make categorical yes-no judgments, since all the depicted stimuli look more or less convincingly transparent.

The present approach was motivated by the empirical findings of Faul (1997). Studying four-region trans- parency, he found that stimuli that looked transparent did not look equally convincingly so. Among the stimuli that looked transparent, some had a special status-namely, those that looked the most convincingly transparent. In Faul's data, these stimuli conformed closely to the strict additive model, whereas the goodness ${ }^{4}$ of the transparency impression decreased monotonically with increasing deviation from the predictions of the strict additive model, until transparency was no longer perceived at all.

It may be instructive to compare the predictions of the present approach with those of Anderson's (1997) model. ${ }^{5}$ According to his model, transparency should be perceived whenever the luminance $\ell$ of the inner elements $P$ is intermediate between the luminance of the outer elements $A$ and the luminance of the background $B$ - that is, whenever

$$
\ell(P)=\alpha \ell(A)+(1-\alpha) \ell(B),
$$

with $0<\alpha<1$. According to the present approach, this is a necessary, but not a sufficient, condition for the perception of transparency. We predicted that among the stimuli satisfying this condition, some should look transparent, whereas others should not, depending on the combination of chromaticities in the stimulus. Furthermore, we predicted that if subjects were instructed to adjust the chromaticity of the inner elements $P$, such that the impression of transparency was the most convincing, they should reliably choose a chromaticity that was consistent with Equation 9. These predictions were tested in Experiment 2. In Experiment 1, we replicated previous findings with purely achromatic stimuli.

\section{EXPERIMENTS}

In order to examine our hypothesis, we performed two experiments. The first was basically a replication of the previously reported findings (van Tuijl \& de Weert, 1979) concerning the luminance conditions for the neon color spreading effect and was performed with achromatic stimuli. The second experiment was performed with chromatic stimuli and was designed (1) to test the hypothesis that configurations satisfying the conditions of the strict additive model look transparent with optimal subjective certainty and (2) to get a first estimate of the limits of the impression of transparency as the deviation from the strict additive model increased.

From the many neon color spreading displays that could potentially have been used for the investigation, a relatively new, animated version of the illusion was chosen, the so-called dynamic neon color spreading display (Cicerone \& Hoffman, 1997; Cicerone, Hoffman, Gowdy, \& Kim, 1995; Hoffman, 1998), because it seemed to produce a slightly more vivid effect, as compared with static displays. Figure 6 schematically shows three frames of such an animation, which can be constructed in the following way. For the first frame, colored dots are randomly or pseudorandomly (Fidopiastis, Hoffman, Prophet, \& Singh, 1998) distributed on a homogenous back- 

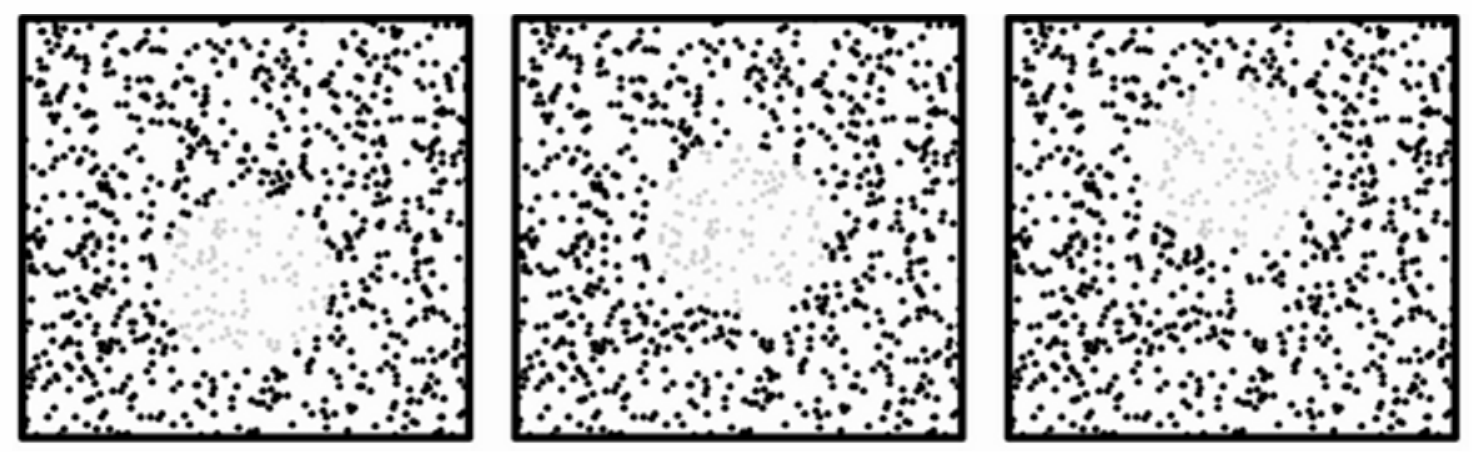

Figure 6. Three frames from an (achromatic) dynamic color spreading display (Hoffman, 1998).

ground. All the dots receive the same color (for instance, black), except the dots located within a virtual circle, which receive another color (for instance, gray). In the next frame, all the dots keep their position; the virtual circle, however, is translated by some small distance, $\Delta x$, and thereupon the colorations of the dots are changed, so that the dots within the circle are all still gray and the dots outside the circle all black. A number of such frames in sequence make up an animation in which a virtual circle moves back and forth at uniform speed. For appropriate choices of colors, one has the impression that the color of the inner dots spreads inside the virtual circle. This neon color (or brightness) spreading is accompanied by a more or less vivid impression of transparency. In regard to their role in producing neon color spreading, the inner dots correspond to the inner cross in the modified Ehrenstein figure, just as the outer dots correspond to the outer segments of the cross. We shall therefore refer to the inner dots as $P$, the outer dots as $A$, and the background as $B$, in analogy to the previous exposition of our model by means of the modified Ehrenstein figure. ${ }^{6}$ The perceptually visible region $Q$ in the modified Ehrenstein figure corresponds to the part of the background inside the virtual circle.

\section{EXPERIMENT 1 Achromatic Stimuli}

This experiment was performed to test our hypothesis with achromatic displays. It is essentially a replication of van Tuijl and de Weert's (1979) investigation using dynamic stimuli instead of static ones. The hypothesis states that the goodness of the transparency impression should depend only on whether the episcotister transmission $\alpha$ is physically possible. However, we also tried to control for other variables that could potentially exert an influence on the transparency impression.

\section{Method}

Procedure and Stimuli. For the special case of achromatic configurations, Equation 9 holds whenever

$$
\ell(P)=\alpha \ell(A)+(1-\alpha) \ell(B)
$$

holds. The strict additive model predicts that transparency is seen when $0<\alpha<1$-that is, when the luminance of the inner dots is intermediate between the luminance of the outer dots and that of the background. The luminance of the inner dots was set according to eight different levels of transmittances $\alpha(-0.4,-0.2,0.2,0.4,0.6$, $0.8,1.2$, and 1.4) for different pairs of luminances for the background and the outer dots. Obviously, the transmittance values $-0.4,-0.2,1.2$, and 1.4 are not physically realizable. As background and outer-dot luminance pairs, all six possible pairs of the three luminances $11.6,23.3$, and $35 \mathrm{~cd} \cdot \mathrm{m}^{-2}$ were used, thus allowing us to check for possible asymmetries resulting from (1) the background's being darker than the outer dots or the converse, (2) differences in the absolute distance between background and outer-dot luminance, and (3) different levels of mean luminance. The three luminances were chosen so as to exploit the monitor gamut maximally. Twenty repetitions of the resulting $8 \times 6$ stimulus conditions, yielding a total of 960 trials, were presented in random order. The subjects were instructed to rate the goodness of the transparency impression on a scale from 0 to 5 . It was emphasized that they were only to express the strength of their subjective confidence that the circular region appeared transparent, as opposed to opaque. They were thus not to rate the degree of transparency in terms of apparent layer density or layer transmittance or to let this influence their judgments. We explicitly instructed the subjects to look for balanced transparency, which was explained to them as the impression that a transparent layer appears in front of the dotted background, through which the latter is seen in its original color. Since pilot experiments had shown that the inner and the outer dots were perceptually indiscriminable at values of $\alpha$ near 1 (an $\alpha$ value equal to 1 implies that they are physically equal), the subjects were allowed to discard the presentation and declare it invalid if this was the case. This explains the unequal number of observations for different $\alpha$ conditions shown in Table 1 . The subjects were allowed to view each presentation as long as they wished. They recorded their judgments, using the "up" and the "down" keys of the keyboard. After they pressed "return," the next presentation immediately followed. Breaks could be made at the subject's own discretion. Each subject finished the experiment in two or three sessions, lasting for a

Table 1

Procentual Proportion of Cases in Which Inner and Outer Dots Were Perceptually Indiscriminable as a Function of $\alpha$

\begin{tabular}{lcccccccc} 
& \multicolumn{8}{c}{$\alpha$} \\
\cline { 2 - 9 } \multicolumn{1}{c}{ Cases } & -0.4 & -0.2 & 0.2 & 0.4 & 0.6 & 0.8 & 1.2 & 1.4 \\
\hline Invalid $(\%)$ & 0 & 0 & 0 & 0 & 0 & 38 & 47 & 6 \\
Valid $(n)$ & 720 & 720 & 720 & 719 & 717 & 445 & 380 & 680 \\
\hline
\end{tabular}


total of approximately $3 \mathrm{~h}$. All of the 7 subjects had normal color vision and normal or corrected-to-normal visual acuity, and all were naive regarding the purposes of the experiment except 3,1 of them being the first author of this paper. The stimuli were presented on a CRT computer monitor, which was carefully calibrated using an LMT C1210 colormeter and controlled by a Cambridge Research Systems VSG 2/3 graphics card, operating in a mode with 8 bits per channel. The 2,500 dots in each presentation had a radius of $1.5 \mathrm{~mm}$ and were distributed pseudorandomly over a region of the monitor measuring $395 \times 270 \mathrm{~mm}$. The (virtual) target disk (radius: $37 \mathrm{~mm}$ ) moved back and forth on a horizontal path, $235 \mathrm{~mm}$ in length, at a constant speed of $10 \mathrm{~cm} / \mathrm{sec}$. A reduction tunnel, fixing the viewing distance to $125 \mathrm{~cm}$, was used to prevent light reflections from the monitor, which was the only source of illumination in the room.

\section{Results}

The mean ratings of transparency goodness as a function of $\alpha$ are shown in Figure 7, which is based on the pooled data from all the subjects except 1 .

This subject failed to see the stimuli with the lighter background as transparent. Visual examination showed that the transparent layers all appear whitish in this kind of stimuli, just like the background, whereas the layers of the other stimuli appear "darkish" or clear like a windowpane. When this subject was shown the stimuli again and asked why he had not rated the stimuli with the light backgrounds as transparent, he stated that he had looked only for clear, windowpane-like transparency.
For the rest of the subjects, ratings were high for $\alpha$ values between 0 and 1 and low for $\alpha$ values outside of this range, as can be seen in Figure 7. This result was predicted by both Anderson's (1997) model and the present model.

In order to check for influences on perceived transparency other than that of $\alpha$, the data were also plotted separately according to the following criteria: (1) whether the difference between the luminances of background and the outer dots was large or small and (2) whether mean luminance was high, medium, or low. Since none of these variables had an influence, these plots are not shown.

One finding from the data that was not explicitly predicted by the model was the moderate transparency ratings at $\alpha=0.8$. However, as was already mentioned, pilot experiments had shown that the outer and the inner dots were rendered perceptually indiscriminable at $\alpha$ values close to one. For this reason, the subjects were given the opportunity to declare such stimuli invalid. As Table 1 shows, the percentage of invalid cases at an $\alpha$ of 0.8 was as high as $38 \%$, suggesting that these stimuli were at the limit of perceptibility, owing to low visible contrast. It is not surprising that the ratings turned out to be a bit lower for these stimuli, since uncertainty is to be expected at the limit of perceptibility and the subjects were supposed to judge the degree of subjective certainty to which the display looked transparent. In summary, the findings of

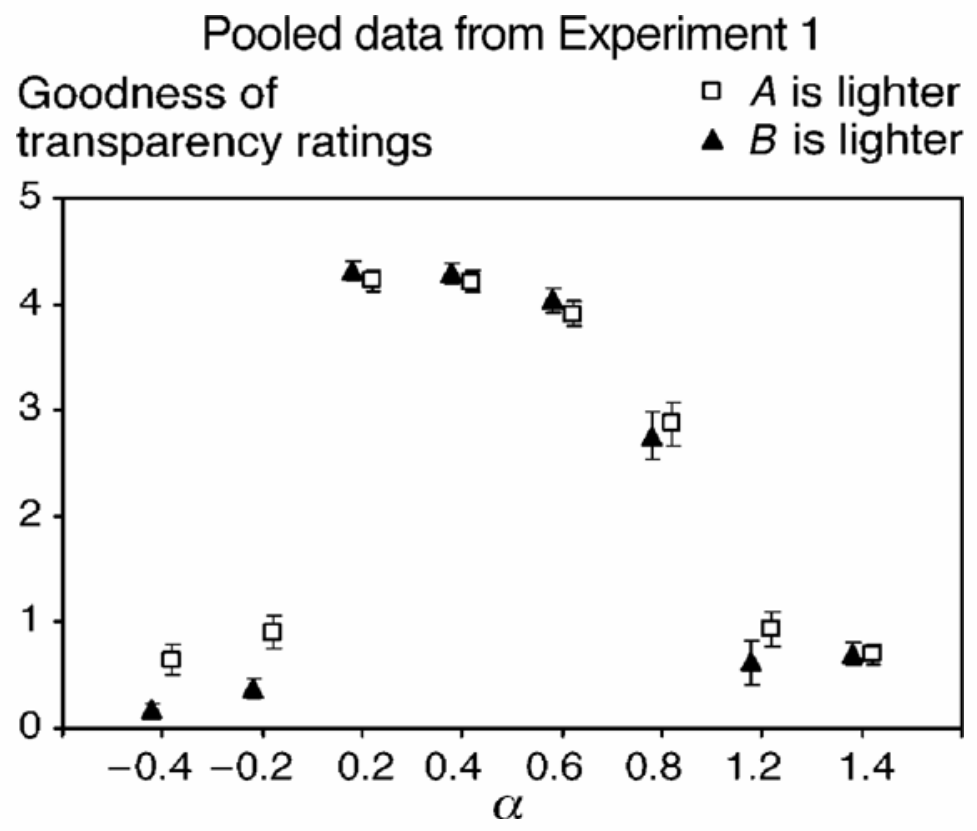

Figure 7. Results from Experiment 1: mean ratings of the subjects' subjective certainty that the configuration looked transparent are given as a function of $\alpha$. According to the model, transparency ratings should be highest for values of $\alpha$ between 0 and 1 . The data are plotted separately for the two levels of the first control variable: outer dots $A$ lighter than background $B$ or the converse. The error bars show the $95 \%$ confidence intervals. 
this experiment are in good agreement with the proposed model.

\section{EXPERIMENT 2 Chromatic Stimuli}

This experiment was performed in order to test our hypothesis for the general case of chromatic stimuli. We wanted to find out whether the proposed model would correctly predict color conditions that made the stimuli look transparent with optimal subjective certainty. Furthermore, we wanted to get a first estimate of the limits of the impression of transparency as the deviation from the strict additive model increased.

\section{Method}

Procedure and Stimuli. The model states that for a given pair of background and outer-dot colors, the set of inner-dot colors producing optimally convincing impressions of transparency is defined by the line segment in color space connecting them (cf. Equation 9). Fixing the luminance of the inner dots to a given value between the luminances of the background and the outer dots is tantamount to defining an equiluminant chromaticity plane in color space, intersecting this line segment at exactly one point, $S$ (see Figure 8). If the subjects had been instructed to adjust the chromaticity of the inner dots inside this equiluminant plane so that the configuration appeared as convincingly transparent as possible, they should have chosen this point $S$, provided the model holds. In order to avoid the usual problems associated with two-dimensional adjustments, such as possible local minima and extremely timeconsuming searches, we restricted the possible settings to a circle in the CIE $1976\left(u^{\prime}, v^{\prime}\right)$-UCS diagram (cf. Wyszecki \& Stiles, 1982, p. 824), in the chosen plane of equiluminance, passing through the predicted intersection point $S$ and centered around the chromaticity of the outer dots.

We used the following four-step procedure for each stimulus. First, the initial chromaticity of the inner dots was randomly set somewhere on the circle. The subject's task was to adjust the chromaticity of the inner dots, using the "left" and "right" keyboard buttons, so that the configuration appeared transparent with optimal subjective certainty. Starting with this optimal chromaticity, the subject's task in the second step was to change the chromaticity of the inner dots in the counterclockwise direction along the circle, by pressing the "left" button, until the configuration did not appear transparent at all. The task in the third step was exactly the same as that in the second step, only now, the chromaticity was to be changed in the opposite direction, starting once again with the chromaticity chosen as optimal in the first step. In the fourth step, the chromaticity chosen as optimal in the first step was reproduced by the computer program, and the subject was instructed to rate the goodness of the transparency impression on a scale from 0 to 5 according to the same criterion as that in Experiment 1. The following information was thus collected. The data recorded in Step 1 allowed us to estimate the point leading to an optimally convincing impression of transparency. The data collected in Steps 2 and 3 provided information about the approximate range of chromaticities leading to a transparency impression. This yields an estimate of how much a configuration can deviate from the optimal point before the phenomenon can no longer be observed at all. The goodness of transparency ratings collected in Step 4 allowed us to verify that the settings in Step 1 actually led to a good impression of transparency and were not merely best choices based only on bad alternatives.

The independent variables were chromaticity and luminance of the background and the outer-dot color pairs and the level of $\alpha$ defined by the point $S$. The levels and combinations of the independent variables were chosen in the following manner. First, we determined the maximal luminance $l_{\max }$ for which a close-to-maximal gamut of chromaticities was still realizable on the monitor used. The two luminances $l_{\max }:=11.3 \mathrm{~cd} \cdot \mathrm{m}^{-2}$ and $l_{\max } / 2$ were used for the background and the outer dots. Then, three chromaticities were sought, lying as far apart as possible but still ensuring that the major parts of the circles, defining the possible settings, were within the monitor gamut. The radii of these circles were, of course, dependent on the choice of the $\alpha$ levels, which were set to $0.2,0.4$, and 0.6. The $\alpha$ level 0.8 was not used, owing to problems of discriminability (see Experiment 1). The background and the outer dots always differed in both chromaticity and luminance. We balanced the role of background and outer dots' luminance, which yielded $2 \times 2$ luminance pairs. This, combined with the three possible (unordered) pairs drawn from the chosen set of three $(x, y)$-chromaticities $\{(0.26,0.18)$, $(0.45,0.31),(0.33,0.37)\}$ and the three $\alpha$ levels, yielded 36 stimuli. Ten repetitions of each stimulus were presented, resulting in a total of 360 presentations for each subject. The subjects were 6 out of the 7 who had participated in Experiment 1-that is, 1 subject did not perform Experiment 2. Again, breaks could be made at the subject's

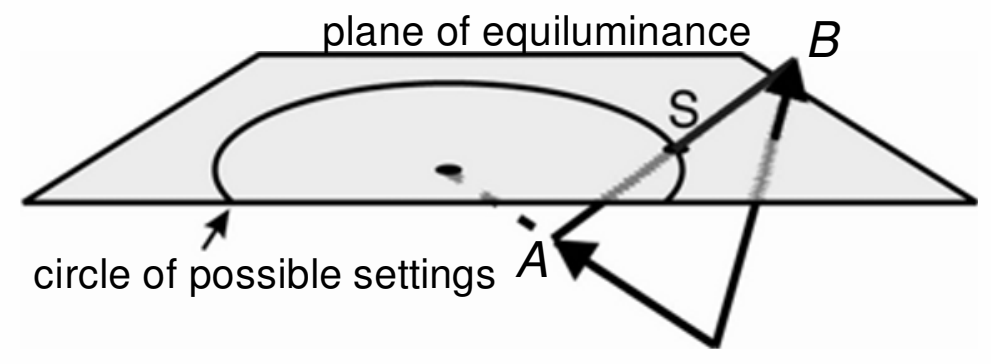

Figure 8. Illustration of the logic used in Experiment 2. Arrows $A$ and $B$ represent possible color codes for the outer dots and the background, respectively. The plane represents the equiluminant chromaticity plane defined by the fixed luminance of the inner dots, $P$. The line connecting $A$ and $B$ represents the set of color codes for the inner dots, $P$, which according to the model, should lead to an optimal impression of transparency. The possible settings were restricted to the depicted circle in the equiluminance plane. The model predicts that the subjects should choose the point of intersection, $S$, between this circle and the line connecting $A$ and $B$. 


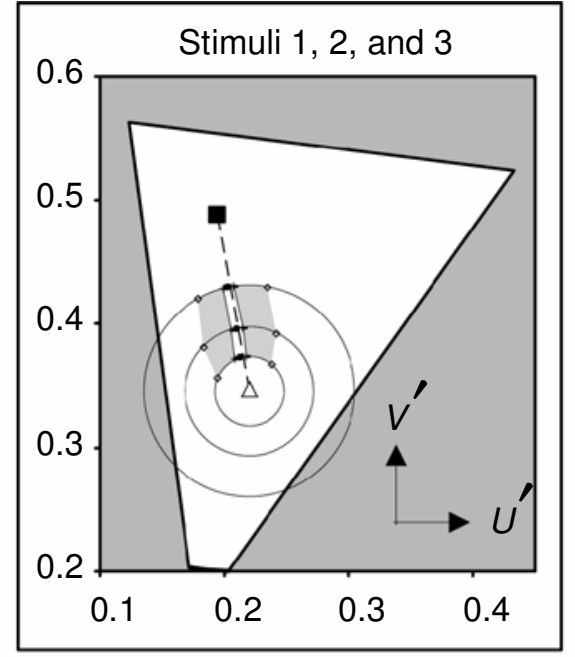

- Chromaticity of the background

$\Delta$ Chromaticity of the outer dots

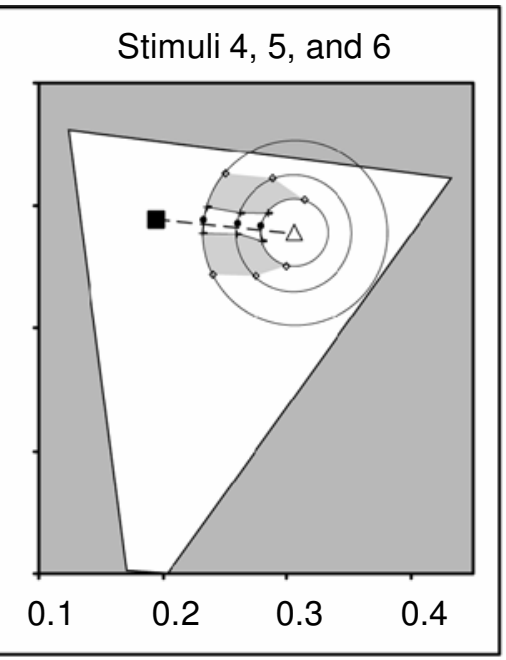

- Chromaticity adjustment for the inner dots

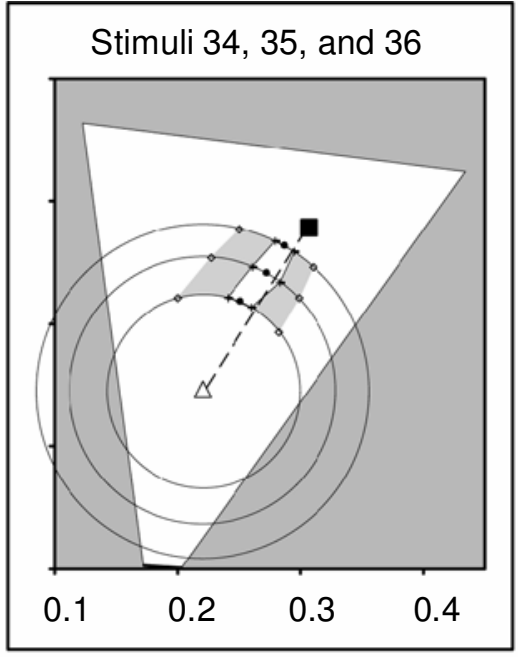

4 Region still seen as transparent

$\triangle$ One standard deviation in each direction

Figure 9. Results from Experiment 2 for nine representative stimuli plotted in the ( $\left.u^{\prime}, v^{\prime}\right)-U$ CS diagram. Background and outer-dot colors were the same for the three stimuli in each plot. The different radii of the circles reflect different levels of $\alpha$. For each of the stimuli, the model prediction is given by the intersection of the circle with the line connecting the background and the outer-dot colors.

own discretion. Each subject finished the experiment in two to four sessions, lasting a total of approximately $6 \mathrm{~h}$. The stimulus configuration and technical data, concerning viewing conditions and apparatus, were identical to those of Experiment 1.

\section{Results}

Some typical data are shown in Figure 9. Each plot in the figure shows the data resulting from three stimulus conditions having the same background and outer-dot colors but different $\alpha$ levels. The inner circle always represents $\alpha=0.2$, the middle circle $\alpha=0.4$, and the outer circle $\alpha=0.6$. The square represents the chromaticity of the background, and the triangle represents the chromaticity of the outer dots. The intersection between the dotted line and a circle represents the optimal point of transparency on this circle, as predicted by the model. The mean ${ }^{7}$ adjustments made for each stimulus condition and pooled over all the subjects are shown as dots. The small white region around the mean settings represents one standard deviation ${ }^{8}$ to each side. The larger shaded region is confined within the means of the adjustments made in Steps 2 and 3 of the four-step procedure. Outside of this region, transparency was no longer seen.

The mean chromaticity adjustments made for the point of optimally convincing transparency were very close to the prediction. The transparency ratings corresponding to these adjustments were close to maximal in all conditions, with a grand mean of 4.09 and a standard deviation of 1.04 .
The right-hand plot in Figure 9 shows the data with the worst fit between data and prediction. This can be verified by reference to Figure 10, which shows the Euclidian distance in $\left(u^{\prime}, v^{\prime}\right)$-coordinates between prediction and mean adjustment for each of the 36 stimulus conditions. The error bars represent the $\left(u^{\prime}, v^{\prime}\right)$ distance between the mean and one standard deviation to each side. The fact that all values are positive reflects merely the fact that distances are always positive. Since the worst fit shown in the right-hand plot of Figure 9 was still very good, it can be stated that the data were very close to the predictions made by the model.

\section{Deviations from the model: all stimuli}

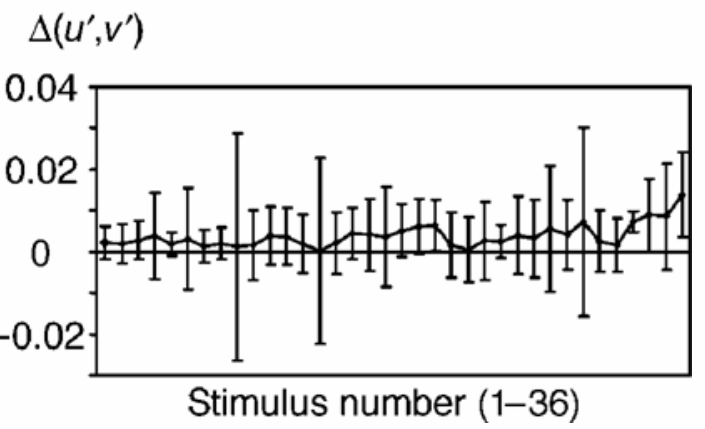

Figure 10. Deviations between prediction and mean setting for all 36 stimuli. $\Delta\left(u^{\prime}, v^{\prime}\right)$ is Euclidian distance in the $\left(u^{\prime}, v^{\prime}\right)$-chromaticity plane. See the text for further details. 


\section{GENERAL DISCUSSION}

In this paper, we adapted an existing model of balanced perceptual transparency, which was originally formulated for patterns consisting of four color regions, to configurations in which only three differently colored regions could be identified. The predictions of the model concerning the color conditions for perceptual transparency in such configurations were tested in two experiments in which dynamic neon color spreading displays were used.

The data of the first experiment, which used achromatic stimuli, showed that convincing impressions of balanced transparency resulted when the luminance of the inner elements was intermediate between the luminances of the outer elements and the background. Stimuli with other luminance relations did not produce convincing impressions of balanced transparency. These results are compatible with both Anderson's (1997) model and the present model. They are, however, seemingly at odds with the data of Bressan (1993b), which showed that balanced perceptual transparency may be observed in some stimuli in which the luminance of the inner elements is not intermediate between the other two luminances in the display. We are not able to give a conclusive explanation of this discrepancy, but it may be noted that the experimental procedure adopted in the present investigation differs from that of Bressan in many respects. For instance, Bressan's subjects were instructed to assign the stimulus to one of four categories, which were found to be possible percepts in a pilot experiment (one of them being the impression of a transparent filter in the region $P \cup Q$ ), whereas in our experiment, the subjects were instructed to report how certain they were that this region looked transparent, using a subjective scale from 0 to 5 . Since the discrepancies between Bressan's and our experimental findings are critical for the evaluation of the validity of her model, on the one hand, and the validity of Anderson's ${ }^{9}$ and the present models, on the other hand, independent replications in which identical stimuli and experimental procedures were used would be of great value.

In Experiment 2, we used chromatic stimuli, which allowed a comparison of the predictions of Anderson's (1997) model with the predictions of the present model. In all of the stimuli used in this experiment, the luminance of the inner elements was intermediate between the two other luminances in the display. Therefore, all possible settings for the chromaticity of the inner elements should have led to the impression of transparency, according to Anderson's model. ${ }^{10}$ Our data clearly show that this is the case only for some combinations of chromaticities, which is to be expected from known models of color transparency (Chen \& D'Zmura, 1998; Da Pos, 1989; D’Zmura et al., 1997; Faul, 1997). Furthermore, it is evident that a real subset of the color combinations that led to an impression of transparency had a special status, since the subjects reliably found a unique setting for each stimulus that led to an impression of transparency that was more compelling than the others. This subset of color combinations is well described by the strict additive model. It may thus be safely concluded that all impressions of transparency are not equally compelling, which is consistent with the findings of similar investigationsin which four-region stimuli were used (Faul, 1997).

A possible interpretation of the present findings is that the color combinations described by the strict additive model led to an impression of balanced transparency, whereas other color combinations, which were also seen as transparent, led to an impression of anomalous, incomplete, or partial transparency (Metelli, Da Pos, \& Cavedon, 1985). Since the subjects of the present experiments were instructed to make settings and ratings for balanced transparency, it is possible that the unique settings of optimally convincing transparency corresponded to cases of balanced transparency, whereas the other color combinations led to impressions of unbalanced transparency, which under this instruction may be seen as transparent, but less convincingly so. Indeed, it can be observed in Figure 5 that the color combinations of the left configurations, which deviate from the strict additive model, lead to impressions of transparency that are not balanced, ${ }^{11}$ whereas the impression in the right configurations - which were intended to fit the strict additive model as far as reproduction allowed it - is rather one of a homogeneous transparent layer through which the grid was seen in its true color.

From the present experiments, it may be concluded that Anderson's (1997) working hypothesis, which maintained that achromatic contrast is the primary determinant of scission, was an oversimplification. However, this does not imply that his general ideas were wrong. It is merely implied that they have to be modified in detail. We hope that the present findings will be helpful in guiding future investigations into the relations between perceptual scission and color illusions.

A point that should be taken into account in future research is that the perception of a transparent layer is at its most convincing in stimuli that differ from those that give the most conspicuous demonstrations of neon color spreading. According to the present approach, this is due to the fact that in stimuli that conform to the additive model and, thus, also look most convincingly transparent, the color (or brightness) spreading has the same perceived chromaticity as the background, whereas in stimuli that are nonoptimal for the perception of balanced transparency, the perceived chromaticity of the spreading is different from that of the background and may, thus, be more noticeable. This important distinction can easily be observed in Figure 5.

\section{REFERENCES}

Anderson, B. L. (1997). A theory of illusory lightness and transparency in monocular and binocular images: The role of contour junctions. Perception, 26, 419-452.

BECK, J., \& IVRY, R. (1988). On the role of figural organization in perceptual transparency. Perception \& Psychophysics, 44, 585-594.

Beck, J., Prazdny, K., \& Ivry, R. (1984). The perception of trans- 
parency with achromatic colors. Perception \& Psychophysics, 35, 407-422.

BRESSAN, P. (1993a). Neon colour spreading with and without its figural prerequisites. Perception, 22, 353-361.

Bressan, P. (1993b). Revisitation of the luminance conditions for the occurrence of the achromatic neon color spreading illusion. Perception \& Psychophysics, 54, 55-64.

Bressan, P., Mingolla, E., Spillmann, L., \& Watanabe, T. (1997). Neon color spreading: A review. Perception, 26, 1353-1366.

Chen, V. J., \& D'Zmura, M. (1998). Test of a convergence model for color transparency perception. Perception, 27, 595-608.

Cicerone, C. M., \& Hoffman, D. D. (1997). Color from motion: Dichoptic activation and a possible role in breaking camouflage. Perception, 26, 1367-1380.

Cicerone, C. M., Hoffman, D. D., Gowdy, P. D., \& Kim, J. S. (1995) The perception of color from motion. Perception \& Psychophysics, 57, 761-777.

DA Pos, O. (1989). Trasparenze. Padua: Icone.

de Weert, C. M. M., \& van Kruysbergen, A. W. H. (1987). Subjective contour strength and perceptual superimposition: Transparency as a special case. In S. Petry \& G. E. Meyer (Eds.), The perception of illusory contours (pp. 165-170). New York: Springer-Verlag.

D’Zmura, M., Colantoni, P., Knoblauch, K., \& Laget, B. (1997). Color transparency. Perception, 26, 471-492.

FAUL, F. (1996). Chromatic scission in perceptual transparency [Abstract]. Perception, 25(Suppl.), 105.

FAUL, F. (1997). Theoretische und experimentelle Untersuchung chromatischer Determinanten perzeptueller Transparenz. Unpublished doctoral dissertation, Christian-Albrechts-Universität, Kiel.

Fidopiastis, C., Hoffman, D. D., Prophet, W., \& Singh, M. (1998). Constructing surfaces and contours in displays of color from motion: The role of nearest neighbors and maximal disks (Memo No. 98-12). Irvine: University of California, Mathematical Behavior Sciences.

Grossberg, S. (1987). Cortical dynamics of three-dimensional form, color, and brightness perception: II. Binocular theory. Perception \& Psychophysics, 41, 117-158.

Hoffman, D. D. (1998). Visual intelligence: How we create what we see. New York: Norton.

KANIZsA, G. (1980). Grammatica del vedere: Saggi su percezione e gestalt [The grammar of sight: Assays on perception and Gestalt]. Bologna: Società Editrice il Mulino.

Metelli, F. (1970). An algebraic development of the theory of perceptual transparency. Ergonomics, 13, 59-66.

Metelli, F., Da Pos, O., \& Cavedon, A. (1985). Balanced and unbalanced, complete and partial transparency. Perception \& Psychophysics, 38, 354-366.

Musatti, C. K. (1953). Luce e colore nei fenomeni del "contrasto simultaneo" della "costanza" e dell' "egualiamento." [Light and color in phenomena of the "simultaneous contrast" of "constancy" and "equalization"]. Archivo di Psicologia, Neurologia e Psichiatria, XIV, 544-577.

Redies, C., \& Spillmann, L. (1981). The neon color effect in the Ehrenstein illusion. Perception, 10, 667-681.

Redies, C., Spillmann, L., \& Kunz, K. (1984). Colored neon and line gap enhancement. Vision Research, 24, 1301-1309.

Schumann, F. (1900). Beiträge zur Analyse der Gesichtswahrnehmungen. Erste Abhandlung: Einige Beobachtungen über die Zusammenfassung von Gesichtseindrücken zu Einheiten. Zeitschrift für Psychologie und Physiologie der Sinnesorgane, 23, 1-32.
Talbot, H. F. (1934). Experiments on light. Philosophical Magazine (3rd series), 5, 321-334

van TuiJL, H. F. J. M. (1975). A new visual illusion: Neonlike color spreading and complementary color induction between subjective contours. Acta Psychologica, 39, 441-445.

van TuiJl, H. F. J. M., \& DE Weert, C. M. M. (1979). Sensory conditions for the occurrence of the neon spreading illusion. Perception, $\mathbf{8}$, 211-215.

VARIN, D. (1971). Fenomeni di contrasto e diffusione cromatica nell' organizzazione spaziale del campo percettivo [Phenomena of contrats and chromatic diffusion in spatial organization of the perceptive field]. Rivista di Psicologia, 65, 101-128.

Watanabe, T., \& Cavanagh, P. (1993). Transparent surfaces defined by implicit $x$ junctions. Vision Research, 33, 2339-2346.

Wyszecki, G., \& StiLes, W. (1982). Color science: Concepts and methods, quantitative data and formulae (2nd ed.). New York: Wiley.

\section{NOTES}

1. It may be appropriate to note that reference to the connection between perceptual scission and Benary's illusion has also been made by Musatti (1953).

2. According to D'Zmura et al. (1997), $\vec{\phi}(E)$ does not necessarily lie within the color cone and may even be the infinitely distant point. This means that the difference vectors may be parallel in this limiting case.

3. In perceptual terms, neon color spreading displays have four differently colored regions, as Bressan (1993b) has noted. It seems natural to regard these perceptual variables as relevant for mechanisms of transparency perception within her theoretical approach. Within our theoretical approach, however, it is natural to refer only to proximal variables.

4. This should not be confused with the perceived degree of transparency - that is, the transmittance of the filter. What is meant is the subjective certainty of the subject that the configuration looks homogeneously transparent, irrespective of the perceived transmittance of the transparent layer.

5. This comparison may not be completely fair to Anderson's (1997) model, since he predicts perceptual scission that may refer both to a decomposition of local luminance into a transparent layer and an underlying surface and to a perceptual decomposition into the reflectance of a surface and the prevailing illumination. In contrast, we only refer to and investigate perceptual transparency.

6. A demonstration of the dynamic color spreading effect is included in Hans Irtel's Color Vision Demonstrations (CVD), which can be downloaded at http://www.uni-mannheim.de/fakul/psycho/irtel/cvd.html.

7. It was computed as the projection of the centroid $\left(\right.$ mean $_{u}$, mean $\left._{v}\right)$ to the circle.

8. The standard deviation was computed as the mean square of the distances between the mean and each adjustment along the circle.

9. Cf. Anderson (1997), p. 448.

10. In fairness, Anderson's (1997) model states only that some kind of perceptual scission should appear in this case, which does not necessarily have to be a scission into transparent layer and background. Still, the comparison is interesting, since the present results would allow a distinction between different kinds of scission.

11. This was also noted by Bressan et al. (1997), p. 1355.

(Manuscript received July 11, 2001; revision accepted for publication January 11,2002.) 